\title{
Primary nasopharyngeal Burkitt's lymphoma in paediatric patients in a non-endemic region: our experience
}

\author{
Santosh K. Swain, Ansuman Sahu, Smrutipragnya Samal \\ Department of Otorhinolaryngology, IMS and SUM Hospital, Siksha "O" Anusandhan University, Kalinganagar, Bhubaneswar, \\ Odisha, India
}

\section{ABSTRACT}

Aim: Burkitt's lymphoma (BL) is an aggressive type of B-cell non-Hodgkin's lymphoma. BL is common in paediatric age in regions where malaria is holoendemic. Its incidence is also high in immunosuppressed patients in non-endemic regions, especially when associated with HIV infection. Primary BL at the nasopharynx in the paediatric age group is extremely rare, especially in non-endemic areas. It often results in misdiagnosis and delayed treatment. BL becomes fatal if untreated or treated late. The aim of this study is to evaluate the clinical presentations, investigations, and treatment of nasopharyngeal BL among paediatric patients in a non-endemic region.

Material and methods: This was a retrospective study in which 21 paediatric patients with nasopharyngeal BL were enrolled, with age under 18 years. This study was done between July 2010 and August 2020.

Results: There were 21 children with nasopharyngeal BL included in this study; 17 male and 4 female, with a male-to-female ratio of $4.25: 1$. The most common presenting symptoms were nasal block (100\%), epistaxis $(71.42 \%)$, rhinorrhoea $(66.66 \%)$, snoring $(57.14 \%)$, and headache $(42.85 \%)$. The most common location of the nasopharynx was the roof of the nasopharynx (28.57\%), lateral wall (23.805), posterior wall (19.04\%), and junction of the roof and posterior wall (28.57\%) of the nasopharynx. All the children were treated with methotrexate, vincristine, cyclophosphamide, doxorubicin, and cytarabine. The mortality of the children with $\mathrm{BL}$ in this study population was related to longer duration of clinical manifestations and also misdiagnosis before getting the final diagnosis of BL.

Conclusions: Paediatric patients with primary nasopharyngeal BL have an excellent survival rate. Early diagnosis and prompt treatment improve the prognosis of nasopharyngeal BL.

\section{KEY WORDS:}

Burkitt lymphoma, nasopharynx, non-endemic region, chemotherapy.

\section{INTRODUCTION}

Burkitt's lymphoma (BL) is a type of NHL, and this malignant neoplasm consists of mature and low-frequency B cells. It constitutes 1 to $3 \%$ of the NHL and is characterized by its aggressive biological behaviour [1]. The least common variety of the lymphoma in all the age groups is usually $\mathrm{BL}$, with an incidence of $1 \%$. In the paediatric age group, $\mathrm{BL}$ is considered as the most frequent variety of NHL with an incidence of 40\% [2]. Burkitt lymphoma (BL) is an aggressive variety of B-cell Non-Hodgkin's lymphoma (NHL) [3]. Burkitt's lymphoma (BL) is a subtype of non-Hodgkin's lymphoma (NHL) and is described as a malignant neoplasm of mature, low-frequency B-cell lymphocytes. BL is broadly divided into 3 major variants: African (endemic) type, American (sporadic) type, and immunodeficiency-associated type [4]. Among them, the African (endemic) form is the commonest variety

\section{ADDRESS FOR CORRESPONDENCE:}

Santosh K. Swain, Department of Otorhinolaryngology, IMS and SUM Hospital, Siksha "O" Anusandhan

University, K8, Kalinganagar, Bhubaneswar - 751003, Odisha, India, e-mail: santoshvoltaire@yahoo.co.in 
worldwide whereas the American (sporadic) form is the commonest type in North America. The sporadic type and immunodeficiency-associated type are commonly found in the abdomen and bone marrow and are rarely seen in head and neck area [5]. The African type of BL is usually associated with Epstein-Barr virus (EBV) and of-

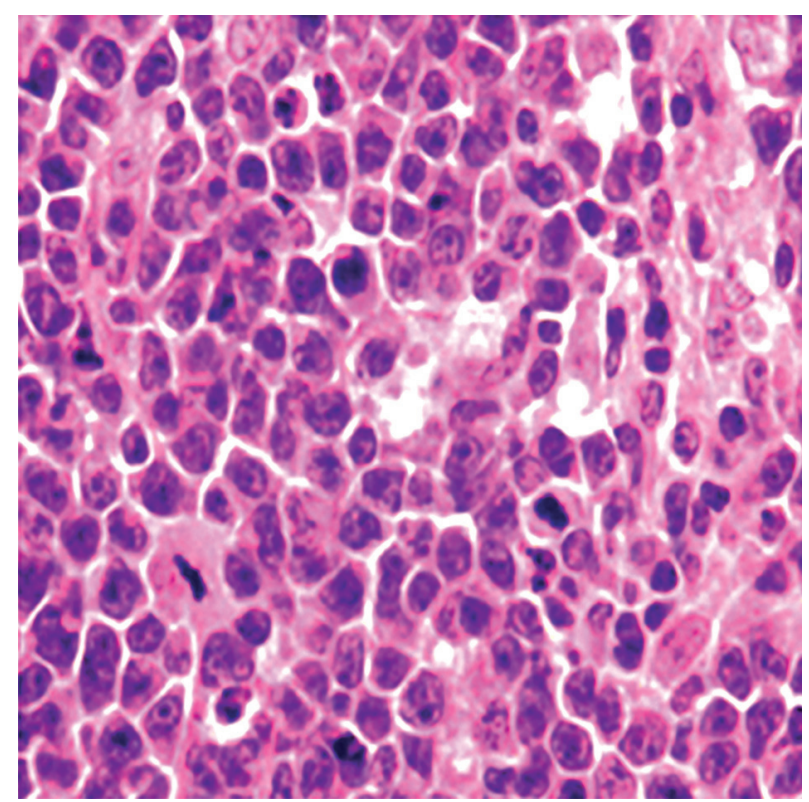

FIGURE. 1. Histopathological picture showing diffuse large B-cells with high mitotic index (HE, 60X)

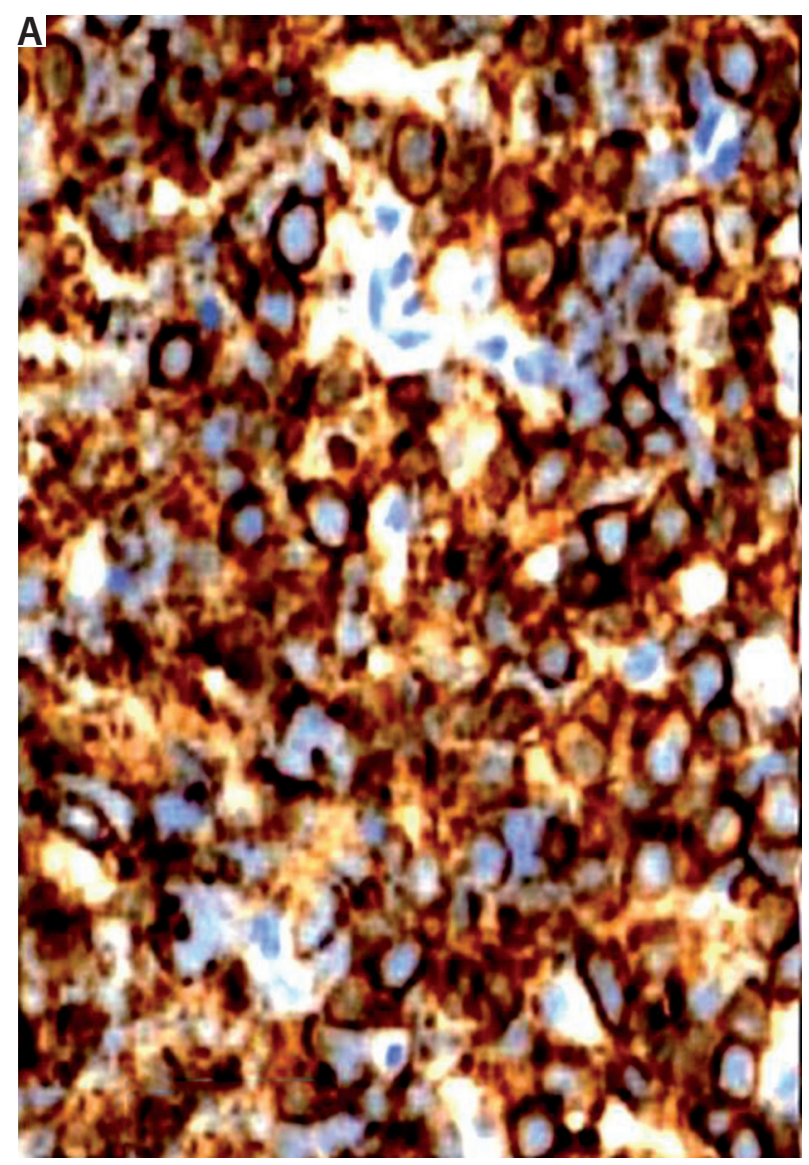

ten affects the facial bones and surrounding soft tissue [6]. Approximately $58 \%$ of BL in head and neck region affect the mandible or maxilla [7]. Because primary nasopharyngeal $\mathrm{BL}$ is rare in the paediatric age group and due to its non-specific clinical presentations, the diagnosis is often delayed or misdiagnosed because of initial thinking of benign aetiologies. Timely and accurate diagnosis of the BL is challenging but crucial. The standard treatment of BL in paediatric age is chemotherapy, but sometimes surgery may be added [8]. In this study, we discuss the clinical profile, management, and outcome of nasopharyngeal BL in paediatric age at a tertiary care teaching hospital in eastern India.

\section{MATERIAL AND METHODS}

This retrospective study was conducted at the Otorhinolaryngology and Paediatric oncology department of a tertiary care teaching hospital in eastern India, based on the data of children and adolescent patients diagnosed with primary nasopharyngeal BL. The only inclusion criteria were patient age below 18 years with nasopharyngeal BL between July 2010 and August 2020. This retrospective study was approved by the Institutional Ethical Committee (IEC) of our medical college with reference number IEC/IMS/SOA/15/22.04.2010. Written consent was obtained from the parents of the participating

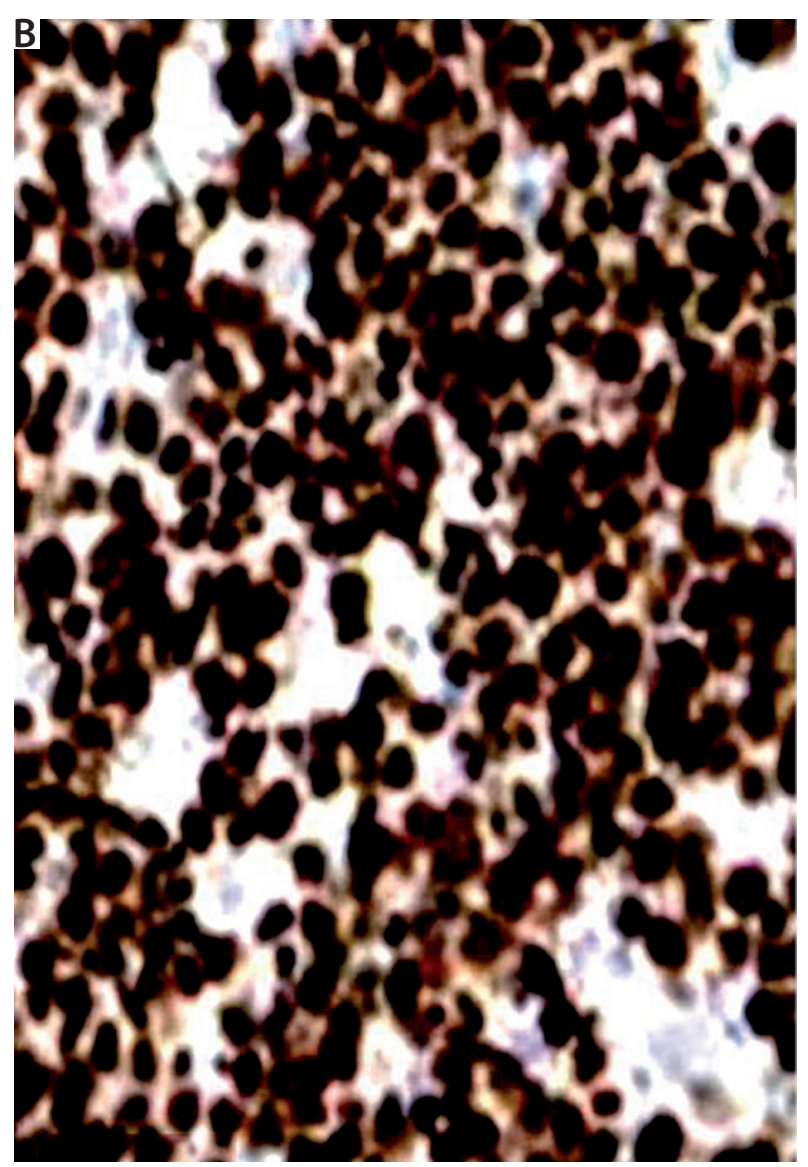

FIGURE. 2A, B. Immunohistological examinations shows strong positive to CD20 (400×) and Ki67 
TABLE 1. Clinical presentations of BL at the nasopharynx

\begin{tabular}{|l|c|c|}
\hline Clinical presentations & Number of patients & Percentage (\%) \\
\hline Nasal obstruction & 21 & 100 \\
\hline Epistaxis & 15 & 71.42 \\
\hline Rhinorrhoea & 14 & 66.66 \\
\hline Snoring & 12 & 57.14 \\
\hline Hyponasal speech & 12 & 57.14 \\
\hline Headache & 9 & 42.85 \\
\hline Otalgia & 7 & 33.33 \\
\hline Hearing loss & 3 & 14.28 \\
\hline Eye pain & 2 & 9.52 \\
\hline
\end{tabular}

children of this study. All children with nasopharyngeal BL underwent a detailed history-taking, a full head and neck examination, systemic examination, and flexible nasopharyngoscopy. The clinical presentations, including their duration, for nasopharyngeal BL such as nasal obstruction, epistaxis, rhinorrhoea, snoring, hyponasal speech, headache, hearing impairment, and pain in the eye were documented. Computed tomography (CT) scan of the neck and nasopharyngoscopy was done routinely in all cases. Magnetic resonance imaging (MRI) was done in all cases to rule out any intracranial and surrounding soft tissue invasion. All the systemic examinations were done in all the participating children at the Paediatric Oncology Department to rule out any systemic involvement. All the participating children underwent biopsy from the nasopharyngeal mass under general anaesthesia, and the diagnosis was confirmed on the basis of the histopathological study (Fig. 1) and immunohistochemical examination (Fig. 2). All the children of nasopharyngeal BL were treated at the Department of Paediatric Oncology. All diagnosed cases of nasopharyngeal BL were treated with 6 cycles of combinations of methotrexate, vincristine, cyclophosphamide, doxorubicin, and cytarabine. The dose of methotrexate was $3 \mathrm{~g} /$ $\mathrm{m}^{2}$ for 3 hours in day one. All the children were followed regularly with physical examination and flexible nasopharyngoscopy every 3 months, every 6 months, and yearly, which indicated the time period after the end of treatment of the individual case.

\section{RESULTS}

There were 21 paediatric patients (19.44\%) diagnosed with primary nasopharyngeal BL out of 108 paediatric registered cases of BL. There were 17 male and 4 female patients, with a male-to-female ratio of 4.25:1. The median age at diagnosis of nasopharyngeal BL was 13 years (range 5-18 years). Eighteen cases (85.71\%) were classified as classical BL, and 3 cases (14.28\%) with pleomorphic lymphomatous cells were classified as atypical or Burkittlike lymphoma. The clinical presentations of the partici-
TABLE 2. Location of the lesions in nasopharyngeal BL

\begin{tabular}{|l|c|c|}
\hline Location at nasopharynx & $\begin{array}{c}\text { Number } \\
\text { of patients }\end{array}$ & $\begin{array}{c}\text { Percentage } \\
\text { (\%) }\end{array}$ \\
\hline Roof of nasopharynx & 6 & 28.57 \\
\hline Lateral wall of the nasopharynx & 5 & 23.80 \\
\hline $\begin{array}{l}\text { Posterior pharyngeal wall } \\
\text { of the nasopharynx }\end{array}$ & 4 & 19.04 \\
\hline $\begin{array}{l}\text { Junction of roof and posterior } \\
\text { wall of the nasopharynx }\end{array}$ & 6 & 28.57 \\
\hline Invading the sphenoid sinus & 4 & 19.04 \\
\hline Invading intracranial space & 3 & 14.28 \\
\hline
\end{tabular}

TABLE 3. Staging of the paediatric BL at the nasopharynx

\begin{tabular}{|l|c|c|}
\hline Staging & Number of patients & Percentage (\%) \\
\hline I & 14 & 66.66 \\
\hline II & 7 & 33.33 \\
\hline III & 0 & 0 \\
\hline IV & 0 & 0 \\
\hline
\end{tabular}

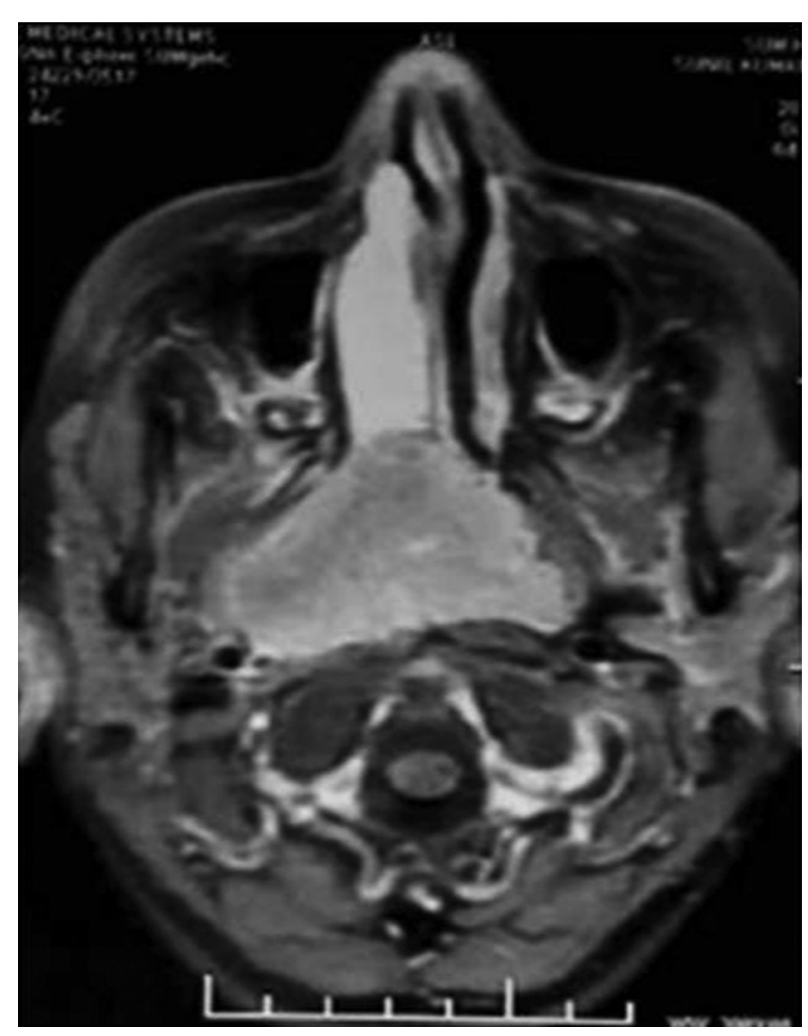

FIGURE. 3. MRI of the head and neck area shows a large nasopharyngeal homogenously enhancing mass with intracranial extension

pating paediatric patients with nasopharyngeal BL were nasal obstruction (100\%), epistaxis (71.42\%), rhinorrhoea (66.66\%), snoring (57.14\%), hyponasal speech (57.14\%), headache (42.85\%), otalgia (33.33\%), hearing impairment (14.28\%), and eye pain (9.52\%) (Table 1). All 3 patients of hearing loss were diagnosed with bilateral serous otitis media. There was no involvement of any cranial nerve, no proptosis, and vision loss in paediatric patients of this 
study. The most common location of the nasopharynx was the roof of the nasopharynx $(28.57 \%)$, the junction of roof and posterior wall (28.57\%), the lateral wall (23.80\%), and the posterior wall (19.04\%) of the nasopharynx (Table 2). There were 4 cases (19.04\%) invading the sphenoid sinus and 3 cases (14.28\%) invading the intracranial part via the foramen ovale (Fig. 3). All the cases were within stage I and II (Ann Arbor staging of the BL). Out of 21 patients, $14(66.66 \%)$ children were with stage I and 7 (33.33\%) were at stage II (Table 3). All the children were treated with methotrexate, vincristine, cyclophosphamide, doxorubicin, and cytarabine. Local radiation therapy was not used for any of the children with nasopharyngeal BL. The mortality of the children with $\mathrm{BL}$ in this study population was related to longer duration of clinical manifestations and also misdiagnosis before obtaining the final diagnosis of BL. After completion of treatment, one patient was lost follow-up for unknown reasons after treatment, without any information to the treating doctor. Two patients $(9.52 \%)$ died after one year of treatment due to relapses of the disease at another site and recurrence at the primary sites. The mortality rate was reported only in 2 cases $(9.52 \%)$ and one patient (4.76\%) lost to follow-up. In this study, the 5-year eventfree survival (EFS) and overall survival (OS) is $90.0 \%$.

\section{DISCUSSION}

Burkitt's lymphoma (BL) is a highly aggressive malignant neoplasm of the B cells. Epidemiologically, BL often affects males, usually children and young adults, constituting approximately $3 \%$ of the cases [9]. The head and neck region is the second most common extranodal location for involvement of the NHL, but the isolated nasopharynx is rarely affected [10]. The common sites of the head and neck region affected by this tumour are part of Waldeyer's ring, orbital adnexal structures, nasal cavity, paranasal sinuses, facial bone salivary glands, and thyroid gland of the head and neck region [11]. The tonsils and nasopharynx were 2 commonly seen extranodal sites in one large American case series of sporadic BL [12]. Extranodal locations of the head and neck region occur in less than $5 \%$ of the patients with sporadic type of $\mathrm{BL}$ [12]. The aetiology of BL is often due to chromosomal translocation $\mathrm{t}(8 ; 14)(\mathrm{q} 24 ; \mathrm{q} 32)$ and deregulation of the C-Myc oncogene [13]. The hallmark genetic aberration as well as the assumed primary event in all 3 epidemiologic subtypes of $\mathrm{BL}$ is $\mathrm{t}(8 ; 14)(\mathrm{q} 24 ; \mathrm{q} 32)$ or its variants $\mathrm{t}(2 ; 8)(\mathrm{p} 12 ; \mathrm{q} 24)$ and $\mathrm{t}(8 ; 22)(\mathrm{q} 24 ; \mathrm{q} 11)$ [13]. All these changes lead to deregulation of the MYC oncogene by its juxtaposition next to one of the enhancer elements in the IGH (14q32), IGK (2p12), or IGL (22q11) locus [13]. However, there are 2 more aetiological factors associated with BL: Epstein-Barr virus infection and malaria. EBV infection and malaria are not exactly aetiological agents, but act as cofactors in the development of BL.
Epstein-Barr virus (EBV) is present in almost all endemic $\mathrm{BL}$, found in $20-30 \%$ of sporadic BL, $40 \%$ of HIV-associated $\mathrm{BL}$, and in a high number of primary immunodeficiency-associated BL or PTLD (post-transplant lymphoproliferative disease). Epstein-Barr virus is known to immortalize B cells and is postulated to provide a block in the apoptotic clearance of B cells with MYC-translocations, thereby helping in clonal evolution. Malaria cooperates with EBV by modulating T-cell response in the pathogenesis of BL [14].

Recent studies on BL have improved the understanding of the pathogenesis of this tumour. Mutations in the transcription factor 3 (TCF3) or its negative regulator ID3 occur in approximately $70 \%$ of sporadic and immunodeficiency-related BL and approximately $40 \%$ of endemic cases. TCF3 promotes the survival and proliferation of the lymphoid cells by activating the B-cell receptor/phosphatidylinositol 3-kinase signalling pathways and modulating the expression of cyclin $\mathrm{D}_{3}$, which is also mutated in approximately $30 \%$ of BL cases [15]. There are varied clinical manifestations in BL. Burkitt's lymphoma is considered as a worrisome clinical condition in which the presenting clinical manifestations may appear as benign. The common clinical manifestations of nasopharyngeal BL are nasal blockage, nasal bleeding, rhinorrhoea, hyponasal speech, facial pain, neck swelling, headache, and otological symptoms like ear pain, fullness in the ear, deafness, and tinnitus. Serous otitis media is a common clinical presentation in any nasopharyngeal mass. The serous otitis media is due to obstruction of the nasopharyngeal opening of the eustachian tube by the BL. The ocular manifestations include eye pain, ptosis, and visual changes. The patient may be presented with visual loss due to involvement of the optic nerve by the tumour mass at the orbital apex. In the case of head and neck BL, the symptoms are jaw swelling, facial swelling, proptosis, and loss of teeth [10]. These symptoms were absent in our study patients. In this study, the commonest clinical symptom was nasal obstruction, which was found in all the participating children (100\%). The presence of red flags like facial palsy, visual loss, and facial swelling warrant prompt investigation [16]. The nasopharyngeal mass is usually biopsied when identified, but not detected at the initial visit. This may be due to benign symptoms such as nasal block/congestion or otalgia often not requiring any invasive examination or procedures such as flexible nasopharyngolaryngoscopy or any imaging in paediatric patients. Hence, the diagnosis may be easily missed or delayed. The rarity of BL in the nasopharynx of the paediatric age patients results in a delay in the diagnosis. Therefore, it is imperative not to disregard simple or benign symptoms and close follow-up and the need for further investigation of non-resolving or recurrent symptoms despite the absence of the red flags. Mortality is usually associated with missed or delayed diagnosis of nasopharyngeal BL. 
Persistent nasal blockage and nasal bleeding require flexible nasopharyngolaryngoscopic examination to find any mass in the nose and nasopharynx. X-ray nasopharynx with lateral view will show the mass at the nasopharynx. Ultrasound of the abdomen and X-ray of the chest in the initial part will provide any evidence of the lesion. Computed tomography (CT) and magnetic resonance imaging (MRI) are 2 important imaging methods that are useful for evaluating the site and extent of the neoplasm. A CT scan will rule out any bony erosion at the nasopharynx and skull base. A CT scan of chest, abdomen, and pelvis can be done to rule out any lesions in the abdomen such liver, kidney, and pancreas. Positron Emission Tomography (PET) scanning is helpful to find any hypermetabolic sites in the body such as head and neck region, chest, abdomen, and pelvis. CT scan, MRI, and PET scan are helpful for a complete work up in paediatric patients of nasopharyngeal BL. Endoscopic biopsy is required for histopathological examination and immunohistochemistry. Because Burkitt's lymphoma spreads so quickly, prompt diagnosis is essential. In the case of persistent nasal blockage with bleeding, primary nasopharyngeal Burkitt's lymphoma can be ruled out quickly by taking a small tissue biopsy from the nasopharyngeal mass. Diagnosis of BL is usually done from harvested tumour tissue: histopathology, immunohistochemistry using a panel of monoclonal antibodies and the molecular method, fluorescent in situ hybridization (FISH) are the currently used approaches for accurate diagnosis of mature B-cell lymphomas (BL, DLBCL, respectively, HGBL - which carries both BL and DLBCL features). Endoscopic biopsy is often done under general anaesthesia in paediatric patients. Incisional biopsy and in situ hybridization are often useful to obtain a correct diagnosis. Histopathological report shows proliferation of large polymorphic $B$ cells with irregular nuclei, suggesting diffuse large B-cell non-Hodgkin's lymphoma. Histologically, the tissue shows a hypercellular area admixed with small to medium, slightly atypical lymphocytes with several tangible body macrophages showing a classical starry sky appearance [17]. Immunohistochemistry is a suitable investigation to identify a specific tumour or cells like anti-CD20 positive and anti-CD3 negative immune-staining tests, which are initially used for corroborating the B lymphocyte origin of the neoplasm and to rule out the T-lymphocyte-originated tumours. On the basis of the pathological findings, the differential diagnosis includes other varieties of high-grade B-cell lymphoma, lymphoblastic lymphoma, leukaemia, and blastoid mantle cell lymphoma [18]. Other high-grade non-lymphomatous childhood malignancies are also considered as differential diagnosis, such as embryonal rhabdomyosarcoma, Ewing's sarcoma, metastatic neuroblastoma, and primitive neuroectodermal tumour (PNET) $[19,20]$. The staging of the $\mathrm{BL}$ is done by using Ann Arbor or more often the St Jude/Murphy staging [21]. At the time of diagnosis approximately $30 \%$ of patients with BL present with early or limited stage of disease (I or II), whereas $70 \%$ of the patients present with widespread stage of disease (stage III or IV) [22]. Patients with clinical variants can spread into the central nervous system and bone marrow.

The different treatment options for BL include surgery, radiotherapy, chemotherapy, and radioimmunotherapy. In general for children, chemotherapy is the primary treatment modality. BL is a highly aggressive disease with a very short doubling time (24-48 hours) and is highly chemo-sensitive. Early initiation of chemotherapy can also resolve emergencies in a short time. The treatment is done according to protocol: short, high-intensity combination chemotherapy is given in therapeutic cycles, based on the classification of patients into risk groups. To classify patients into individual risk groups, the clinical stage, tumour respectability, tumour mass size based on lactate dehydrogenase value and the presence of lactate dehydrogenase, and the presence of bone marrow and / or CNS involvement are assessed. The treatment of $\mathrm{BL}$ includes intermittent high-dose chemotherapy, and common chemotherapeutic agents are cyclophosphamide, vincristine, doxorubicin, methotrexate, and cytarabine [23, 24]. The role of surgery in BL is still controversial. The role of surgery is limited to the following: 1. taking a representative tissue sample for histological examination, or 2. to tumour extirpation, if possible, but without performing a mutilation procedure. The respectability of the tumour may place the patient in a lower risk group and thus allow the intensity of chemotherapy to be reduced. Extensive surgery postpones the possibility of starting chemotherapy. The debulking of the tumour is usually beneficial when done with permissive anatomical location, although its role in the treatment of the head and neck region has not been well studied [25]. In the case of poor prognosis of the BL, loco-regional radiotherapy plays an important role in giving local symptomatic relief [26]. Burkitt's lymphoma dramatically responds to chemotherapies inducing regressions of the tumour and often leading to longterm remission [25]. The chemotherapy is the mainstay of the treatment in nasopharyngeal BL. Radiotherapy is not used in children with BL. The remission rate is up to $90 \%$ for 2 years, reported with an intensive chemotherapy regimen [28]. Rituximab, a monoclonal CD20 antibody, has also been used with promising results when used with a typical chemotherapeutic regimen $[29,30]$. The prognosis of BL patients treated with highly active antiretroviral drugs and chemotherapy are considered satisfactory. The mortality and morbidity of these patients are often associated with delays or misdiagnosis and associated infections such as bacterial sinusitis, reactive lymphadenitis, maxillary alveolar abscess, adenotonsillar hypertrophy, and otitis media with effusion. Presently 5-year survival for advanced BL in paediatric patients and young adults have increased 2 - to 3 -fold in the last 3 decades, from $85 \%$ to $90 \%$ with less than 6 months of intensive 
chemotherapy [31]. This is due to a better understanding of the disease biology and advancement of chemotherapeutic drugs.

\section{CONCLUSIONS}

Primary Burkitt's lymphoma at the nasopharynx in the paediatric age group is uncommon. Patients of primary BL at nasopharynx often present with nonspecific and insidious symptoms ranging from nasal obstruction to headache to hearing loss. Otolaryngologists and paediatricians must remain vigilant of this haemato-oncological entity at the nasopharynx. Burkitt's lymphoma in paediatric age is usually aggressive but treatable. Burkitt's lymphoma is an aggressive neoplasm and often considered as a serious health condition, so prompt and appropriate treatment of the disease is always required.

\section{DISCLOSURE}

The authors declare no conflict of interest.

\section{REFERENCES}

1. Kissi L, El Bouihi R, Lamchahab M, et al. Burkitt's lymphoma of the oral cavity: about a case. Pan Afr Med J 2017; 26: 63.

2. Thomas AG, Vaidhyanath R, Kirke R, et al. Extranodal lymphoma from head to toe: part 1, the head and spine. AJR Am J Roentgenol 2011; 197: 350-356.

3. Brillantino C, Rossi E, Minelli R, et al. Burkitt lymphoma in children: An unusual presentation. Front Med Case Rep 2020; 1: 1-8.

4. Bouda GC, Traoré F, Couitchere L, et al. Advanced Burkitt Lymphoma in Sub-Saharan Africa Pediatric Units: Results of the Third Prospective Multicenter Study of the Groupe Franco-Africain d'Oncologie Pédiatrique. J Glob Oncol 2019; 5: 1-9.

5. Huang S, Suhrland MJ, Gritz D. Recurrent immunodeficiency-associated Burkitt lymphoma presenting as severe anterior uveitis. Ocul Oncol Pathol 2015; 2: 62-65.

6. Zhang W, Yang J, Zhou C, et al. Early response observed in pediatric patients with relapsed/refractory Burkitt lymphoma treated with chimeric antigen receptor T cells. Blood 2020; 135: 2425-2427.

7. Márquez Moyano JA, Mohamed Youssef AS, Sánchez Gutiérrez R, et al. Linfoma de Burkitt nasofaríngeo en edad pediátrica. Acta Otorrinolaringol Esp 2004; 55: 295-297.

8. Urbano S, López E, Harris J. Immunodeficiency-associated Burkitt's lymphoma in pediatric patients: a clinical case report. J Oral Res 2018; 7: 141-144.

9. Huang S, Suhrland MJ, Gritz D. Recurrent immunodeficiency-associated Burkitt lymphoma presenting as severe anterior uveitis. Ocul Oncol Pathol 2015; 2: 62-65.

10. Amusa YB, Adediran IA, Akinpelu VO, et al. Burkitt's lymphoma of the head and neck region in a Nigerian tertiary hospital. West Afr J Med 2005; 24: 139-142.

11. Vega F, Lin P, Medeiros LJ. Extranodal lymphomas of the head and neck. Ann Diagn Pathol 2005; 9: 340-350.

12. Rodriges-Fernandes CI, Perez-de-Oliveira ME, Arboleda LP, et al. Clinicopathological analysis of oral Burkitt's lymphoma in pediatric patients: A systematic review. Int J Pediatr Otorhinolaryngol 2020; 134: 110033 .
13. Paneal RI, Love CL, Shingleton JR, et al. The whole genome landscape of Burkitt lymphoma subtypes. Blood 2019; 134: 1598-1607.

14. Hutcheson RL, Chakravorty A, Sugden B. Burkitt lymphomas evolve to escape dependencies on Epstein-Barr virus. Front Cell Infect Microbiol 2021; 10: 606412.

15. Swerdlow SH, Campo E, Pileri SA, et al. The 2016 revision of the World Health Organization classification of lymphoid neoplasms. Blood 2016; 127: 2375-2390.

16. Hong X, Khalife S, Bouhabel S, et al. Rhinologic manifestations of Burkitt Lymphoma in a pediatric population: Case series and systematic review. Int J Pediatric Otorhinolaryngol 2019; 121: 127-136.

17. Dunleavy K. Approach to the diagnosis and treatment of adult Burkitt's lymphoma. J Oncol Pract 2018; 14: 665-671.

18. Enrique Rendón-Macías M, Alfonso Valencia-Ramón E, Fajardo-Gutiérrez A. Clinical and epidemiological characteristics of Burkitt lymphomas in pediatric patients from two defined socioeconomic regions in Mexico. J Trop Pediatr 2017; 63: 253-259.

19. Swain SK, Samal S, Mohanty JN, et al. Nasopharyngeal carcinoma among the pediatric patients in a non-endemic region: Our experiences at a tertiary care teaching hospital in Eastern India. Egyptian Pediatric Association Gazette 2020; 68: 1-6.

20. Swain SK, Samal S, Sahu MC. Nasopharyngeal carcinoma in a six year old female child: a case report. Pediatr Pol 2019; 94: 132-135.

21. Kasamon YL, Swinnen LJ. Treatment advances in adult Burkitt lymphoma and leukemia. Curr Opin Oncol 2004; 16: 429-435.

22. Grande BM, Gerhard DS, Jiang A, et al. Genome-wide discovery of somatic coding and noncoding mutations in pediatric endemic and sporadic Burkitt lymphoma. Blood 2019; 133: 1313-1324.

23. Ferry JA. Burkitt's lymphoma: clinicopathologic features and differential diagnosis. The Oncologist 2006; 11: 375-383.

24. Rosolen A, Perkins LS, Pinkerton CR, et al. Revised international pediatric non-Hodgkin lymphoma staging system. J Clin Oncol 2015; 33: 2112-2118.

25. Brigger MT, Cunningham MJ. Malignant cervical masses in children. Otolaryngol Clin North Am 2015; 48: 59-77.

26. Lowry L, Smith P, Qian W, et al. Reduced dose radiotherapy for local control in non-Hodgkin lymphoma: A randomized phase III trial. Radiother Oncol 2011; 100: 86-92.

27. AI Burshaid DI, Nasser MA, Nagaraj V, et al. Sporadic Burkitt's lymphoma of the hypopharynx: A case report. Saudi J Med Med Sci 2019; 7: 114-117.

28. Wu K, Majumdar S, Bull PD. Blocked nose and snoring in a 6-yearold boy. Int J Pediatr Otorhinolaryngol 2004; 68: 597-560.

29. Barnes JA, LaCasce AS, Feng Y, et al. Evaluation of the addition of rituximab to CODOX-M/IVAC for Burkitt's lymphoma: a retrospective analysis. Ann Oncol 2011; 22: 1859-1864.

30. Alexander S, Ferrando AA. Pediatric lymphoma. In: Nathan and Oski's hematology and oncology of infancy and childhood. Orkin $\mathrm{SH}$ et al. (eds.). $8^{\text {th }}$ ed. Philadelphia: Elsevier Saunders, 2015; 16481668.

31. de Leval L, Hasserjian RP. Diffuse large B-cell lymphomas and Burkitt lymphoma. Hematol Oncol Clin North Am 2009; 23: 791-827. 